\title{
AVALIAÇÃO ISOCINÉTICA DA FADIGA EM JOGADORES DE FUTEBOL PROFISSIONAL
}

MS. FERNANDA SEGANFREDO WEBER

Mestre em Ciências do Movimento Humano pela Universidade Federal do Rio Grande do Sul (UFRGS), Laboratório de Pesquisa do Exercício (LAPEX/EsEF/UFRGS)

(Porto Alegre - Rio Grande do Sul - Brasil)

E-mail: fefeweber@hotmail.com

MS. BRUNA GONÇALVES CORDEIRO DA SILVA

Mestre em Ciências do Movimento Humano pela Universidade Federal do Rio Grande do Sul (UFRGS), Laboratório de Pesquisa do Exercício (LAPEX/EsEF/UFRGS)

(Porto Alegre - Rio Grande do Sul - Brasil)

E-mail: brugcs@hotmail.com

\section{DR. EDUARDO LUSA CADORE}

Doutor em Ciências do Movimento Humano pela Universidade Federal do Rio Grande do Sul (UFRGS), Laboratório de Pesquisa do Exercício (LAPEX/EsEF/UFRGS)

(Porto Alegre - Rio Grande do Sul - Brasil)

E-mail: edcadore@yahoo.com.br

\section{MS. STEPHANIE SANTANA PINTO}

Mestre em Ciências do Movimento Humano pela Universidade Federal do Rio Grande do Sul (UFRGS), Laboratório de Pesquisa do Exercício (LAPEX/EsEF/UFRGS)

(Porto Alegre - Rio Grande do Sul - Brasil)

E-mail: tetisantana@yahoo.com.br

\section{DR. RONEI SILVEIRA PINTO}

Professor Adjunto do Departamento de Educação Física da Escola de Educação Física da Universidade Federal do Rio Grande do Sul (EsEF/UFRGS), Doutor em Ciências do

Desporto pela Universidade Técnica de Lisboa (Portugal) Laboratório de Pesquisa do Exercício (LAPEX/EsEF/UFRGS)

(Porto Alegre - Rio Grande do Sul - Brasil)

E-mail: ronei.pinto@ufrgs.br

\begin{abstract}
RESUMO
O objetivo deste estudo foi revisar os resultados a respeito da relação entre a fadiga, o desempenho esportivo e a incidência de lesões no futebol, bem como verificar, nos estudos citados, os protocolos de teste de fadiga mais utilizados nesse esporte. Para isso, foram selecionadas as bases de dados SCOPUS, PubMed, Journals Ovid, Web of Science e BMJ e as palavras-chave: soccer and isokinetic, soccer and fatigue test, soccer and fatigue and isokinetic e soccer and injury. Os estudos incluídos nesta revisão foram selecionados pelo título
\end{abstract}


contendo as palavras-chave e pela leitura do resumo, verificando se havia uma relação entre o futebol, os testes de avaliação de fadiga, o desequilíbrio muscular e a lesão em jogadores profissionais. A literatura apresenta diversos protocolos de avaliação isocinética da fadiga em jogadores profissionais de futebol. Embora não exista um consenso sobre qual é o melhor protocolo de avaliação, o índice de fadiga é um importante parâmetro a ser determinado. Além disso, as razões musculares, sobretudo a razão funcional, permitem inferências sobre o equilíbrio muscular e a propensão ao desenvolvimento de lesões relacionadas à articulação do joelho em jogadores de futebol.

PALAVRAS-CHAVE: Fadiga; razão funcional; protocolos isocinéticos; futebol.

\section{INTRODUÇÃO}

O futebol é um esporte que tem várias competições anuais, por vezes mais de uma ocorre ao mesmo tempo, repercutindo em uma carga elevada de trabalho para os atletas. Devido a isso, os jogadores profissionais devem estar sempre preparados fisicamente para obterem bom desempenho nas partidas.

Na prática do futebol, os membros inferiores são muito solicitados para a realização de ações como: corridas, sprints, saltos, chutes e desarmes (COMETTI et al., 200 I). O músculo quadríceps femoral possui um papel importante na execução dos saltos, chutes e passes, enquanto que os ísquiotibiais controlam as atividades de corrida e estabilizam o joelho nas situações que envolvem mudanças de direção ou desarme do adversário. Ressalta-se que esses últimos atuam também no momento de passe de bola ou chute, a partir da contração excêntrica (CARVALHO; CABRI, 2007). Sendo assim, as habilidades específicas do futebol dependem da produção de força desses músculos (COMETTI et al., 200 I; CARVALHO; CABRI, 2007).

A fadiga muscular é uma falha na manutenção de força ou potência requerida ou esperada (SANGNIER; TOURNY-CHOLLET, 2007). Ela vai se pronunciando no decorrer do jogo de futebol principalmente no quadríceps femoral e ísquiotibiais, pois esses são os grupos musculares mais utilizados na realização das ações do futebol. É importante dar atenção à fadiga, pois quando o músculo diminui a força e a potência, ele não consegue mais realizar os movimentos com tanta habilidade e precisão (RAMPININI et al., 2009; STONE; OLIVER, 2009; THORLUND; AAGAARD; MADSEN, 2009), e o risco de lesão também aumenta devido a desequilíbrios musculares que se acentuam (GREIG, 2008; SMALL et al., 2008). Visto que a fadiga parece influenciar aspectos importantes do jogo de futebol, é fundamental que ela seja avaliada e a resistência a ela treinada.

Existem vários testes de fadiga muscular e, entre eles, alguns que utilizam o dinamômetro isocinético como instrumento de medida. Ainda não existe um consenso sobre a melhor forma de avaliar a fadiga da musculatura anterior e posterior da coxa em jogadores de futebol. Alguns autores realizam testes isocinéticos 
de flexão e extensão de joelho antes e após protocolos de exercícios exaustivos específicos de futebol, em laboratório (MAGALHÃES et al., 200।; RAHNAMA et al., 2003; GREIG, 2008; SMALL et al., 2008), e outros somente realizam os testes isocinéticos com grande número de repetições e velocidade angular média ou elevada (KAWABATA et al., 2000; GOULARTet al., 2007; SANGNIER; TOURNY-CHOLLET, 2007; SANGNIER; TOURNY-CHOLLET, 2008; WEBER et al., 20 I 0). Sendo assim, o objetivo do presente estudo foi revisar os resultados a respeito da relação entre a fadiga com o desempenho e a incidência de lesões no futebol, bem como verificar, nos estudos citados, os protocolos de teste de fadiga nesse esporte.

\section{METODOLOGIA}

Para realização da presente revisão, foi realizada uma metabusca no portal Periódicos Capes, utilizando como área do conhecimento as ciências da saúde e subárea, a educação física e esportes. Foram selecionadas as seguintes bases de dados: SCOPUS, PubMed, Journals Ovid, Web of Science e BMJ e as seguintes palavras-chave foram utilizadas: soccer and isokinetic, soccer and fatigue test, soccer and fatigue and isokinetict, soccer and injury.

Os estudos incluídos neste estudo foram selecionados primeiramente pelo título contendo as palavras chave e, posteriormente, pela leitura do resumo, verificando se havia uma relação entre o futebol, os testes de avaliação de fadiga em jogadores profissionais, o desequilíbrio muscular e a lesão. Desta forma, foram incluídos 37 artigos científicos para a realização deste estudo de revisão.

\section{FUTEBOL}

O futebol é um esporte complexo de contato que demanda habilidades físicas, fisiológicas, técnicas e táticas (ANDERSEN et al., 2004). É um dos esportes mais praticados no mundo. Em 2003, cerca de 250 milhões de jogadores licenciados em 204 países foram registrados na FIFA, e aproximadamente I\% participava em níveis profissionais (ANDERSEN et al., 2003). No Brasil, a maioria dos jogadores profissionais participa de mais de um campeonato no decorrer do ano, por vezes alguns se sobrepõem, repercutindo em uma carga elevada de trabalho para os atletas. Devido a isso, os jogadores profissionais devem estar sempre preparados fisicamente para obterem bom desempenho nas partidas e se prevenirem de possíveis lesões.

\section{FADIGA}

A fadiga é a falha na manutenção de força ou potência requerida ou esperada (SANGNIER; TOURNY-CHOLLET, 2007). As causas da fadiga durante o exercício 
muscular incluem fatores que residem no cérebro (mecanismos centrais), bem como nos músculos (mecanismos periféricos) (DAVIS, 1995). A fadiga central se refere a processos mais proximais, e pode ser definida como uma falha da ativação voluntária do músculo induzida pelo exercício progressivo, ela pode ser atribuída a mecanismos supra-espinais (TAYLOR; GANDEVIA, 2008). Já a fadiga de origem periférica é atribuída a alterações na função da junção neuromuscular, atividade elétrica das fibras musculares e níveis metabólitos musculares (HOLDER-POWELL; JONES, 1990).

\section{FADIGA E DESEMPENHO DAS HABILIDADES}

Durante o jogo de futebol, os músculos quadríceps femoral e ísquiotibiais são muito solicitados para a realização de ações como: corridas, sprints, saltos, chutes e desarmes (COMETTI et al., 200I). Como essas ações ocorrem com grande frequência durante as partidas oficiais, esses músculos acabam entrando em fadiga.

A fadiga que ocorre durante a partida de futebol tende a diminuir o desempenho das habilidades motoras específicas e aumentar o risco de lesão. Com relação à diminuiçãa do desempenho esportivo, Stone e Oliver (2009) mostraram em seu estudo que 45 min de exercícios específicos de futebol não causam diminuição no desempenho de sprints, porém causam reduções significativas na performance das habilidades específicas do futebol. Rampinini et al. (2009) realizaram um estudo através de análise de partidas, gravado em vídeo, de jogadores de futebol italiano, e concluíram que durante o segundo tempo de jogo há um declínio significativo, tanto do desempenho físico quanto das habilidades técnicas (envolvimentos com a bola, passes curtos e sucesso em passes curtos). Além disso, no estudo de revisão de Reilly, Drust e Clarke (2008), foi verificado que os jogadores de futebol europeus parecem correr mais durante o primeiro tempo de jogo em comparação ao segundo.

Com esses resultados, pode-se inferir que a fadiga causada pelo declínio na força muscular máxima e capacidade de força muscular rápida, pode afetar o desempenho funcional em ações como corridas, mudanças de direção, passes e chutes.

\section{FADIGA E LESÃO}

A incidência de lesão é relativamente alta no futebol, sendo que a cada I .000 horas de jogo ocorrem cerca de 17 a 24 lesões. Alguns estudos mostraram um índice ainda mais elevado, chegando a 53 lesões em 1.000 horas (RAHNAMA et al., 2003). Além disso, essas lesões ocorrem mais durante as partidas do que nos treinos, sendo sua incidência 4-6 vezes mais alta nos jogos em comparação aos treinos (JUNGE; DVORAK, 2004).

As lesões mais comuns que ocorrem em atletas do futebol são entorses, distensões e contusões, e afetam predominantemente o tornozelo, as articulações 
do joelho e os músculos da coxa e panturrilha (CHOMIAK et al., 2000; WOODS et al., 2004). Dentre essas, as mais frequentes são de ligamento cruzado anterior e distensão dos ísquiotibiais (GREIG, 2008).

As lesões de não-contato são aquelas que ocorrem sem haver contato ou pancada com outro jogador. A fadiga causada pelo jogo, assim como os desequilíbrios musculares e articulares podem aumentar o risco da ocorrência dessas lesões. $\bigcirc$ percentual de lesões de não-contato no futebol é de $26 \%$ a 59\%, e elas ocorrem principalmente durante corridas ou mudanças de direção, aterrissagem de um salto, extensão de joelho quase completa e chutes (JUNGE; DVORAK, 2004; GELI et al., 2009). Ressalta-se que a maioria desses movimentos envolve os ísquiotibiais, atuando principalmente de forma excêntrica, e que 47\% das distensões dos ísquiotibiais ocorreram durante os últimos minutos do segundo tempo (WOODS et al., 2004), o que parece ter uma relação direta com a fadiga.

Rahnama et al. (2002) observaram, em um estudo, que o risco de lesões durante uma partida de futebol é maior nos primeiros e últimos 15 min de jogo. Nos primeiros minutos, o risco surge pelo intenso engajamento no início da partida, todavia, nos últimos 15 min, as ações de risco de lesão moderada surgem como resultado da fadiga muscular.

A fadiga muscular no futebol é resultado de 90 min de sprints, deslocamentos variados, passes, saltos, chutes, enfim, movimentos intensos e repetitivos utilizando os membros inferiores. Esses movimentos solicitam principalmente os músculos quadríceps femoral e ísquiotibiais. Estudos mostram que a constituição das fibras nesses dois grupos musculares é diferente, os ísquiotibiais apresentam maior quantidade de fibras rápidas em comparação ao quadríceps e essas fibras são mais fatigáveis. Dessa forma, os ísquiotibiais parecem perder mais força do que o quadríceps ao longo de vários movimentos consecutivos intermitentes (SANGNIER; TOURNY-CHOLLET, 2007; GREIG, 2008; SANGNIER; TOURNY-CHOLLET, 2008).

Para movimentos de chute, aterrissagem de salto e frenagem de corridas, os ísquiotibiais possuem um papel fundamental de realizar contração excêntrica para frear o movimento do membro, estabilizando então o segmento da coxa e preservando o joelho de possíveis lesões. Sendo assim, esse músculo tem grande importância para os atletas do futebol e a sua avaliação, assim como o aumento da resistência à fadiga dos ísquiotibiais é fundamental.

\section{RAZÕES MUSCULARES E PROTOCOLOS DE AVALIAÇÃO ISOCINÉTICA DA FADIGA}

Uma das formas de se avaliar o equilíbrio muscular entre o quadríceps femoral e os ísquiotibiais pode ser a partir de um teste realizado em um equipamento 
chamado dinamômetro isocinético. A avaliação da força muscular especificamente realizada em dinamômetro isocinético vem sendo utilizada no diagnóstico de disfunções neuromusculares, na reabilitação, no treino, e na investigação, como indicador da função e desempenho de certos grupos musculares, sendo a sua medição feita a partir do pico de torque (PT) (DVIR, 2002). Os principais desequilíbrios avaliados são aqueles de PT entre o membro dominante e não dominante (diferença bilateral) e dos músculos antagonistas em relação aos agonistas (BROWN, 2000; MAGALHÃES et al., 200 I; CARVALHO; CABRI, 2007).

Croiser et al. (2008) afirmam que o esporte de alto nível faz com que a repetição de um gesto estereotipado e a especificidade do treinamento ocasione modificações frequentes da força muscular. Além disso, os mesmos autores explicam que as adaptações concernem regularmente os músculos agonistas pela atividade condicionada ao nível da performance, tanto que seus antagonistas, não solicitados, ficam pouco desenvolvidos, e que a prevenção dos desequilíbrios musculares representa uma questão pertinente. No caso do futebol, o maior cuidado seria com os flexores, que realizam um grande trabalho durante o jogo, mas parecem ser pouco treinados fora do mesmo.

\section{RAZÕES MUSCULARES}

Por muitos anos, os desequilíbrios musculares relacionados ao joelho vêm sendo avaliados a partir da razão convencional (PT concêntrico dos flexores/ PT concêntrico dos extensores), e valores entre 0,5 e 0,7 são considerados dentro da normalidade (DVIR, 2002). Estudos prévios, usando a razão convencional do joelho, demonstraram que uma razão menor de 0,6 avaliada a $60 \%$ s representa $77,5 \%$ de probabilidade de lesão no joelho em atletas de elite de futebol (DAUTY et al., 2003). $\bigcirc$ uso somente dessa razão, como indicadora de desequilíbrios, apresenta limitações, pois a contração concêntrica dos ísquiotibiais simultânea à contração concêntrica do quadríceps é uma situação que não ocorre durante a execução de movimentos funcionais.

No movimento de chutar a bola, por exemplo, ocorre a contração concêntrica dos extensores do joelho e excêntrica dos flexores; logo, a comparação dos picos de torque durante os referidos tipos de contração, seria o mais indicado para a observação dos desequilíbrios na articulação do joelho em jogadores de futebol. Sendo assim, a chamada razão funcional (PT excêntrico dos flexores / PT concêntrico dos extensores) é atualmente utilizada para tal avaliação, e valores próximos de I são considerados dentro da normalidade (AAGAARD et al., 1998; TOURNY-CHOLLET et al., 2000; COOMBS; GARBUTT, 2002; CROSIER et al., 
2008; IGA et al., 2008). Curiosamente, Crosier et al. (2008) observaram que os atletas de futebol com razão funcional maior que I,4 nunca haviam sofrido lesão de ísquiotibiais. Isso nos faz refletir sobre o valor de I como normativo, pois parece que durante o jogo, devido à fadiga, há uma queda no PT dos músculos da coxa, fazendo com que essa razão mude e fique baixa, a ponto de colocar a articulação do joelho em risco.

\section{PROTOCOLOS DE AVALIAÇÃO ISOCINÉTICA DE FADIGA}

A resistência à fadiga e a habilidade do músculo de recuperar-se durante exercícios intermitentes de alta intensidade são atributos importantes da função neuromuscular, e têm sido associados à maturação biológica, sexo e nível de treinamento. A resistência à fadiga é maior em crianças e pré-puberes, do que em adultos do sexo masculino, e semelhante em mulheres comparadas a homens em todas as faixas etárias (DIPLA et al., 2009).

O índice de fadiga, avaliado através do teste de fadiga no dinamômetro isocinético, indica a habilidade do indivíduo de realizar o mesmo movimento, repetidas vezes, com uma determinada resistência. $\mathrm{Na}$ avaliação isocinética, ele é analisado levando em consideração a diferença dos picos de torque entre as primeiras e as últimas repetições realizadas durante o teste. Ainda não existe um consenso para a melhor forma de avaliação isocinética de fadiga em flexores e extensores de joelho especificamente para atletas de futebol. Algumas pesquisas utilizaram somente o dinamômetro isocinético para verificar a fadiga, todavia, outras, além do dinamômetro, realizam também circuitos ou outros protocolos adicionais. É possível visualizar esses dois tipos de pesquisas nos Quadros I e 2, respectivamente. Além disso, pode-se observar no Quadro 3, o percentual de fadiga de extensão e flexão concêntrica do joelho encontrados nos diferentes protocolos.

Os protocolos, somente no dinamômetro (QUADRO I), têm como vantagem o tempo reduzido de avaliação, porém não há garantia que a fadiga reproduzida neste equipamento seja igual ou semelhante àquela que o jogador terá durante o jogo de futebol. Entretanto, os protocolos utilizando deslocamentos (QUADRO 2), reproduzem melhor a fadiga em condições de jogo, porém são mais longos e somente utilizam deslocamentos, deixando de fora as demais habilidades do futebol, como passes, chutes, mudanças de direção e saltos, e que também demandam força dos membros inferiores. 
Quadro I. Protocolos utilizando somente o dinamômetro isocinético

\begin{tabular}{|c|c|c|c|c|}
\hline Autores & População & Séries & Repetições & Velocidade \\
\hline Kawabata et al. (2000) & $\begin{array}{l}\text { Jogadores de futebol, } \\
\text { beisebol e marato- } \\
\text { nistas }\end{array}$ & $\begin{array}{l}3(\mathrm{i}: 10 \\
\min )\end{array}$ & 50 & $180 \% \mathrm{~s}$ \\
\hline $\begin{array}{l}\text { Sangnier e Tourny-Chollet } \\
(2007 ; 2008)\end{array}$ & Jogadores de futebol & I & 50 & $180^{\circ} / \mathrm{s}$ \\
\hline $\begin{array}{l}\text { Goulart, Dias e Altimari } \\
\text { (2007) }\end{array}$ & Jogadores de futebol & I & 30 & $300 \% / \mathrm{s}$ \\
\hline $\begin{array}{l}\text { Wrigth, Ball e } \\
\text { Wood (2009) }\end{array}$ & $\begin{array}{l}\text { Jogadores de futebol } \\
\text { recreativos }\end{array}$ & I & 50 & $120 \% \mathrm{~s}$ \\
\hline
\end{tabular}

Legenda: i, intervalo entre as séries.

Quadro 2. Protocolos utilizando deslocamentos e dinamômetro isocinético

\begin{tabular}{|c|c|c|c|c|c|}
\hline \multirow[b]{2}{*}{ Autores } & \multirow[b]{2}{*}{ População } & \multirow[b]{2}{*}{ Protocolo I } & \multicolumn{3}{|c|}{ Protocolo 2 - dinamômetro } \\
\hline & & & Séries & $\begin{array}{l}\text { Repeti- } \\
\text { ções }\end{array}$ & Velocidade \\
\hline $\begin{array}{l}\text { Rahnama et al. } \\
\text { (2003) }\end{array}$ & $\begin{array}{l}\text { Jogadores } \\
\text { de futebol }\end{array}$ & $\begin{array}{l}\text { Esteira - reproduzin- } \\
\text { do as velocidades da } \\
\text { partida de futebol } \\
\text { (90 min) }\end{array}$ & I & 3 & $\begin{array}{l}60 \% / \mathrm{s} \\
120 \% \mathrm{~s} \\
300 \% \mathrm{~s}\end{array}$ \\
\hline $\begin{array}{l}\text { Magalhães et al. } \\
(200 I)\end{array}$ & $\begin{array}{l}\text { Jogadores } \\
\text { de futebol }\end{array}$ & $\begin{array}{l}\text { LIST ( } 5 \text { vezes) } \\
\text { caminhadas }(3 \times 20 \mathrm{~m}) \text {, } \\
\text { sprints }(1 \times 20 \mathrm{~m}) \text {, } \\
\text { recuperação }(4 \mathrm{~s}) \text {, } \\
\text { velocidades de corrida } \\
\text { correspondendo a } \\
55 \% \text { e depois } 95 \% \text { do } \\
\mathrm{VO}_{2 \text { máx }}(3 \times 20 \mathrm{~m})\end{array}$ & I & 3 & $90 \% / \mathrm{s}$ \\
\hline Greig (2008) & $\begin{array}{l}\text { Jogadores } \\
\text { de futebol }\end{array}$ & $\begin{array}{l}\text { Esteira - reproduzin- } \\
\text { do as velocidades da } \\
\text { partida de futebol } \\
\text { (90 min) }\end{array}$ & $\begin{array}{l}1 \\
1 \\
1\end{array}$ & $\begin{array}{l}5 \\
5 \\
5\end{array}$ & $\begin{array}{l}60 \% / \mathrm{s} \\
180^{\circ} / \mathrm{s} \\
300^{\circ} / \mathrm{s}\end{array}$ \\
\hline Small et al. (2008) & $\begin{array}{l}\text { Jogadores } \\
\text { de futebol }\end{array}$ & $\begin{array}{l}\text { Circuito - reproduzin- } \\
\text { do as velocidades da } \\
\text { partida de futebol (STAF } \\
90 \mathrm{~min} \text { ) }\end{array}$ & । & 3 & $120 \% \mathrm{~s}$ \\
\hline
\end{tabular}

Legenda: LIST, teste intermitente de movimento de Loughborough 
Quadro 3. Percentual de fadiga de extensão e flexão concêntrica do joelho encontrados nos diferentes protocolos

\begin{tabular}{|c|c|c|c|c|c|}
\hline \multicolumn{3}{|c|}{ Protocolo somente isocinético } & \multicolumn{3}{|c|}{ Protocolo de deslocamentos + isocinético } \\
\hline \multirow[b]{2}{*}{ Autor } & \multicolumn{2}{|l|}{ \% Fadiga } & \multirow[b]{2}{*}{ Autor } & \multicolumn{2}{|l|}{ \% Fadiga } \\
\hline & $\begin{array}{l}\text { Extensores } \\
\text { (mín-máx) }\end{array}$ & $\begin{array}{l}\text { Flexores } \\
\text { (mín-máx) }\end{array}$ & & $\begin{array}{l}\text { Extensores } \\
\text { (mín-máx) }\end{array}$ & $\begin{array}{l}\text { Flexores } \\
\text { (mín-máx) }\end{array}$ \\
\hline $\begin{array}{l}\text { Kawabata et al. } \\
(2000)\end{array}$ & $46-48$ & $35-40$ & $\begin{array}{l}\text { Rahnama } \\
\text { et al. (2003) }\end{array}$ & $8-15$ & $15-17$ \\
\hline $\begin{array}{l}\text { Sangnier e } \\
\text { Tourny-Chollet } \\
\text { (2007) }\end{array}$ & $54-59$ & $58-67$ & Greig (2008) & Sem mudança & Sem mudança \\
\hline $\begin{array}{l}\text { Goulart, Dias e } \\
\text { Altimari (2007) }\end{array}$ & $69-77$ & $65-72$ & $\begin{array}{l}\text { Small } \\
\text { et al. (2008) }\end{array}$ & Sem mudança & Sem mudança \\
\hline
\end{tabular}

Legenda: mín= valor mínimo, máx= valor máximo.

Com relação aos resultados dos estudos, pode-se observar que os protocolos com deslocamentos reproduzindo o jogo de futebol apresentaram menor diminuição de torque em comparação àqueles que utilizaram somente o dinamômetro isocinético (Quadro 3). Eles também se assemelharam aos resultados de Thorlund et al. (2009), principalmente para extensores de joelho ( I 1 \%) após a realização de uma partida de futebol. A fadiga apresentada pelos flexores foi menor após o jogo, em comparação aos protocolos de fadiga. Ainda, pode ser claramente observado no Quadro 3, que os valores dos percentuais de fadiga são muito diferentes entre os protocolos somente isocinéticos, e aqueles que envolvem também o deslocamento. Dessa maneira, não é possível ou adequado realizar comparações entre estudos, se estes utilizam diferentes protocolos para avaliar a fadiga.

Magalhães et al. (200 I) verificaram o impacto do teste intermitente de movimento de Loughborough (LIST) de deslocamento versus o jogo de futebol analisando vários fatores, entre eles parâmetros neuromusculares. Não foram encontradas diferenças nos resultados após o jogo ou LIST, dessa forma, para os parâmetros neuromusculares de PT, o teste parece ter reproduzido os efeitos de um jogo de futebol amigável. Sabe-se que um jogo de futebol amigável, apesar de apresentar os mesmos movimentos do jogo de futebol profissional, não pode ser comparado a esse. No jogo profissional existem vários fatores que tornam a partida diferente, os jogadores normalmente possuem mais preparo físico, habilidade técnica e tática, também há mais influências internas psicológicas e externas da torcida, entre outras. Dessa forma, conclui-se que esse protocolo com deslocamentos ainda pode ser aprimorado para reproduzir um jogo de futebol profissional. 
Sangnier e Tourny-Chollet (2007) concluíram que o declínio de PT dos flexores foi significativamente maior que o dos extensores, após as primeiras 15 repetições para a perna dominante, e após 40 repetições para a perna não dominante. Em outro estudo semelhante a esse, utilizando o mesmo protocolo, Sangnier e Tourny-Chollet (2008) investigaram a perda de força dos ísquiotibiais e quadríceps, para estabelecer um modelo de regressão usando o menor número de flexões e extensões durante um teste de fadiga. Eles concluíram que, para estabelecer um modelo linear, seria necessário no mínimo 20 extensões e I 5 flexões para verificar perdas no PT. Porém, para verificar o decréscimo na razão com um modelo quadrático, seriam necessárias 25 repetições dos movimentos.

Esses estudos mostraram que, durante o teste isocinético de fadiga, a resistência à fadiga diverge entre o quadríceps femoral e os ísquiotibiais, sendo que, em alguns deles, os flexores reduzem mais o torque em comparação aos extensores (SANGNIER; TOURNY-CHOLLET, 2007). Essa maior diminuição de PT dos ísquiotibiais pode ser atribuída a características estruturais e metabólicas dos mesmos, visto que há uma maior proporção de fibras do tipo II nesses músculos, em relação ao quadríceps (GARRETT et al., 1984). Como as fibras do tipo II fadigam mais rapidamente (MAUGHAN; GLEESON; GREENHAFF, 2000), os ísquiotibiais apresentam maior diminuição de PT ao longo do tempo. Curiosamente, há pesquisas que mostram o contrário, sendo a fadiga do quadríceps femoral maior, e isso poderia ocorrer pelo menor preparo físico desses músculos pelos atletas.

Devido a essa queda diferenciada de PT entre ísquiotibiais e quadríceps, causada pela fadiga, evidencia-se uma tendência ao desequilíbrio muscular. Oliveira et al. (2008) realizaram um estudo em não-atletas, medindo o PT concêntrico e excêntrico dos flexores e extensores de joelho, antes e após exercícios contínuos de corrida. Eles observaram que a razão convencional permaneceu a mesma após o exercício, porém a razão funcional diminuiu significativamente após a corrida. O mesmo resultado foi obtido no estudo de Greig (2008), porém, Sangnier e Tourny-Chollet (2007) observaram diminuição, tanto na razão funcional quanto na convencional. Essas condições de diminuição diferenciada de PT entre os músculos anteriores e posteriores da coxa podem afetar a estabilidade articular e predispor a articulação do joelho às lesões. Dessa forma, sugere-se que a razão convencional para atletas fique acima da média de 0,66 e a razão funcional maior de I, visto que, durante a partida, devido às condições de fadiga progressiva, ela poderá decair, aumentando o risco de lesão muscular. 
De acordo com a presente revisão, pode-se inferir que o futebol é um esporte que demanda uma grande exigência dos músculos quadríceps femoral e ísquiotibiais durante sua realização, o que resulta na fadiga dos mesmos. A fadiga ocasiona uma redução no desempenho do futebol, bem como pode estar associada à incidência de lesões nesse esporte. A literatura apresenta diversos protocolos de avaliação de fadiga em jogadores profissionais de futebol. Embora não exista um consenso sobre qual é o melhor protocolo de avaliação, o índice de fadiga é um importante parâmetro a ser determinado. Além disso, tem sido sugerido que as razões musculares, sobretudo a razão funcional, permitem inferências sobre o equilíbrio muscular e a propensão ao desenvolvimento de lesões relacionadas à articulação do joelho em jogadores de futebol.

\section{Isokinetic evaluation of fatigue in professional soccer players}

ABSTRACT: The aim of this study was to review the results of the relation between fatigue with the performance and incidence of injuries in soccer, as well as to check in the mentioned studies the fatigue test protocols in this sport. For this, were selected SCOPUS, PubMed, Journals Ovid, Web of Science e BMJ databases, and the keywords soccer and isokinetic, soccer and fatigue test, soccer and fatigue and isokinetic, soccer and injury. The studies included in this study were selected by the title containing the keywords and by reading the abstract, then checking if there was a relation between soccer, evaluation of fatigue in professional players, muscle imbalance and injury. The literature presents several protocols for the assessment of fatigue in professional soccer players. Although there is no consensus on what is the best protocol for evaluation, the fatigue index is an important parameter to be determined. Furthermore, the muscle ratios, especially the functional ratio, allow inferences about muscle balance and the propensity to develop related injuries to the knee joint in soccer players.

KEYWORDS: Fatigue; functional ratio; isokinetic protocol; soccer.

\section{Evaluación isocinética de fatiga en futbolistas profesionales}

RESUMEN: El objetivo de este estudio fue revisar los resultados sobre la relación entre la fatiga con el rendimiento y la incidencia de lesiones en fútbol, así como verificar en los estudios citados, los protocolos de ensayo de fatiga en este deporte. Para eso, se seleccionaron las bases de datos SCOPUS, PubMed, Journals Ovid, Web of Science e BMJ y las palabras clave soccer and isokinetic, soccer and fatigue test, soccer and fatigue and isokinetic, soccer and injury. Los estudios incluidos en este estudio fueron seleccionados por el título conteniendo las palabras clave y mediante la lectura del resumen, verificando se trataba de la relación entre el fútbol, la evaluación de la fatiga en jugadores profesionales, el desequilibrio muscular y la lesión. La literatura presenta varios protocolos para la evaluación de la fatiga en los jugadores de fútbol. Aunque no hay consenso sobre cuál es el mejor protocolo para la evaluación, el índice de fatiga es un parámetro importante que se determine. Además, las razones musculares, sobre todo la razón funcional, permite inferencias sobre el equilibrio muscular y la propensión a desarrollar lesiones relacionadas con la articulación de la rodilla en atletas.

PALABRAS CLAVE: Fatiga; razón funcional; protocolos isocineticos; fútbol. 


\section{REFERÊNCIAS}

AAGAARD, P. et al. A new concept for isokinetic hamstring: quadriceps muscle strength ratio. American Journal of Sports Medicine, Chicago, v. 26, n. 2, p. 231-237, mar. 1998.

ANDERSEN, T. et al. Football incident analysis: a new video based method to describe injury mechanisms in professional football. British Journal of Sports Medicine, Loughborough, v. 37, n. 3, p. 226-232, june 2003.

ANDERSEN, T. et al. Video analysis of injuries and incidents in Norwegian professional football. British Journal of Sports Medicine, Loughborough, v. 38, n. 5, p. 626-63 I, oct. 2004. BROWN, L. Isokinetics in human performance. Champaign: Human Kinetics, 2000. 456p.

CARVALHO, P; CABRI, J. Avaliação isocinética da força dos músculos da coxa dos futebolistas. Revista Portuguesa de Fisioterapia no Desporto, São Domingos de Rana, v. I, n. 2, p. 4- I2, jul. 2007.

CHOMIAK, J. et al. Severe injuries in football players. Influencing factors. American Journal of Sports Medicine, Chicago, v. 28, suppl. 5, p. S58-S68, sept. 2000.

COMETTI, G. et al. Isokinetic strength and anaerobic power of elite, subelite and amateur French soccer players. International Journal of Sports Medicine, New York, v. 22, n. I, p. 4451 , jan. 2001 .

COOMBS, R.; GARBUTT, G. Developments in the use of the hamstring/quadriceps ratio for the assessment of muscle balance. Journal of Sports Science and Medicine, Bursa, v. I, n. 3, p. 56-62, sept. 2002.

CROSIER, J. L. et al. Strength imbalances and prevention of hamstring injury in professional soccer players: A prospective study. American Journal of Sports Medicine, Chicago, v. 36, n. 8 p. |469-1475, aug. 2008.

DAUTY, M. et al. Identification of previous hamstring injury by isokinetic concentric and eccentric torque measurement in elite soccer players. Isokinetics and Exercise Science, Amsterdan, v. II, n. 3, p. I34- |44, sept. 2003.

DAVIS, J. M. Central and peripheral factors in fatigue. Journal of Sports Sciences, London, v. 13, ed. especial, p. S49-S53, july 1995.

DIPLA, K. et al. Fatigue resistance during high-intensity intermittent exercise from childhood to adulthood in males and females. European Journal of Applied Physiology, Heildelberg, v. I06, n. 5, p. 645-653, apr. 2009.

DVIR, Z. Isocinética: avaliações musculares, interpretações e aplicações clínicas. São Paulo: Manole, 2002. 201 p.

GARRETT, W. E. et al. Histochemical correlates of hamstring injuries. American Journal of Sports Medicine, Chicago, v. 12, n. 2, p. 98-103, mar. 1984. 
GELI, E. A. et al. Prevention of non-contact anterior cruciate ligament injuries in soccer players. Part I: Mechanisms of injury and underlying risk factors. Knee Surgery, Sports Traumatology, Arthroscopy, New York, v. 17, n. 7, p. 705-729, june. 2009.

GREIG, M. The Influence of soccer-specific satigue on peak isokinetic torque production of the knee flexors and extensors. American Journal of Sports Medicine, Chicago, v. 36, n. 7, p. | 403- | 409, july 2008.

GOULART, L. F. et al. Força isocinética de jogadores de futebol categoria sub-20: comparação entre diferentes posições de jogo. Revista Brasileira de Cineantropometria e Desempenho Humano, Florianópolis, v. 9, n. 2, p. 165-169, abr. 2007.

HOLDER-POWELL, H. M.; JONES, D. A. Fatigue and muscular activity. Physiotherapy, Oxford, v. 76, n. II, p. 672-679, dec. 1990.

IGA, J. et al. Cross-sectional investigation of indices of isokinetic leg strength in youth soccer players and untrained individuals. Scandinavian Journal of Medicine and Science in Sports, Oxford, v. 19, n. I, p. I-6, oct. 2009.

JUNGE, A.; DVORAK, J. Soccer injuries: a review on incidence and prevention. Sports Medicine, Auckland, v. 34, n. 13, p. 929-938, dec. 2004.

KAWABATA, Y. et al. Measurement of fatigue and knee flexor and extensor muscle. Acta Medica Okayama, Okayama, v. 54, n. 2, p. 85-90, apr. 2000.

MAGALHÃES, J. et al. Avaliação isocinética da força muscular de atletas em função do desporto praticado, idade, sexo e posições específicas. Revista Portuguesa de Ciência do Desporto, Porto, v. I, n. 2, p. 13-21, jan./jun. 2001.

MAUGHAN, R. et al. Bioquímica do exercício e do treinamento. São Paulo: Manole, 2000. 240p.

OLIVEIRA, A. S. C. et al. Heavy-intensity aerobic exercise affects the isokinetic torque and functional but not conventional hamstrings: quadriceps ratios. Journal of Electromyography and Kinesiology, Oxford, v. 19, n. 6, p. 1079-1084, dec. 2008.

RAHNAMA, $N$. et al. Muscle fatigue induced by exercise simulating the work rate of competitive soccer. Journal of Sports Sciences, London, v. 21, n. II , p. 933-942, nov. 2003.

RAHNAMA, N. et al. A. Injury risk associated with playing actions during competitive soccer. British Journal of Sports Medicine, Londres, v. 36, n. 5, p. 354-356, oct. 2002.

RAMPININI, E. et al. Technical performance during soccer matches of the Italian serie A league: effect of fatigue and competitive level. Journal of Science and Medicine in Sport, Maryland Heights, v. 12, n. I, p. 227-233, nov. 2009.

REILLY, T. et al. Muscle fatigue during football match-play. Sports Medicine, Auckland, v. 38, n. 5, p. 357-367, may 2008.

SANGNIER, S; TOURNY-CHOLLET, C. Effect of fatigue on hamstrings and quadriceps during isokinetic fatigue testing in semiprofessional soccer players. International Journal of Sports Medicine, New York, v. 28, n. I I, p. 952-957, nov. 2007. 
SANGNIER, S.; TOURNY-CHOLLET, C. Study of the fatigue curve in quadriceps and hamstrings of soccer players during isokinetic endurance testing. Journal of Strength and Conditioning Research, Philadelphia, v. 22, n. 5, p. I458-1467, sept. 2008.

SMALL, K. et al. The effects of multidirectional soccer-specific fatigue on markers of hamstring injury risk. Journal of Science and Medicine in Sport, Maryland Heights, v. I3, n. I, p. 120-125, jan. 2010.

STONE, K. J.; OLIVER, J. L. The effect of 45 minutes of soccer-specific exercise on the performance of soccer skills. International Journal of Sports Physiology and Performance, Champaign, v. 4, n. 2, p. 163-175, june 2009.

TAYLOR, J. L.; GANDEVIA, S. C. A comparison of central aspects of fatigue in submaximal and maximal voluntary contractions. Journal of Applied Physiology, Bethesda, v. I04, n. 2, p. 542-550, ago. 2008.

THORLUND J. B. et al. Rapid muscle force capacity changes after soccer match play. International Journal of Sports Medicine, New York, v. 30, n. 4, p. 273-278, apr. 2009.

TOURNY-CHOLLET, C. et al. Isokinetic knee muscle strength of soccer players according to their position. Isokinetics and Exercise Science, Amsterdam, v. 8, n. 4, p. 187-193, oct. 2000.

WEBER, F.S. et al. Avaliação isocinética em jogadores de futebol profissional e comparação do desempenho entre as diferentes posições ocupadas no campo. Revista Brasileira de Medicina do Esporte, São Paulo, v. I6, n. 4, p. 264-268, jul./ago. 2010.

WOODS, C. et al. The football association medical research programme: an audit of injuries in professional football: analysis of hamstring injuries. British Journal of Sports Medicine, Londres, v. 38, n. I, p. 36-4I, feb. 2004.

WRIGHT, B.; BALL, N.; WOOD, L. Fatigue, H/Q ratios and muscle coativation in recreational football players. Isokinetics and Exercise Science, Amsterdam, v. 17, n. 3, p. I61 - 167, july 2009.

Recebido: II jun. 2010

Aprovado: 9 set. 201 I

Endereço para correspondência:

Fernanda Seganfredo Weber

Rua Felizardo, 750 (LAPEX/EsEF/UFRGS)

Bairro Jardim Botânico

Porto Alegre-RS

CEP: $90690-200$ 